\title{
Imaging ability and visual processing of EEG waveforms
}

\author{
BENJAMIN WALLACE \\ Cleveland State University, Cleveland, Ohio \\ and \\ THOMAS F. COLLURA \\ Cleveland Clinic Foundation, Cleveland, Ohio
}

\begin{abstract}
Individual differences in the reading and processing of EEG waveforms were examined. Neurologists were asked to read an EEG printout while a video camera recorded their activity in processing the printout. Two strategies were evident in reading the printouts. For those who used a detail strategy, reading of printouts was slow and often relied on many referrals back to previously examined patterns. Those who used a holistic strategy read printouts quickly and did not exhibit the back-and-forth referral activity of the previous group. The implications of these individual differences for future applications in the processing of EEGs are discussed.
\end{abstract}

There is a considerable amount of evidence to indicate that individual differences exist with respect to the use of imagery in the completion of a cognitive task (see Wallace, 1991). For example, Wallace (1988) presented subjects with a list of objects that were embedded within a pictorial scene. Subjects were then presented the scene and were requested to locate the objects. In addition, prior to participation in the visual search task, subjects were assessed on their imaging ability on the Vividness of Visual Imagery Questionnaire (VVIQ; Marks, 1973). Subjects who scored in the top one third of this test (vivid imagers) performed the visual search differently from those who scored in the bottom one third (poor imagers).

Vivid imagers spent a significantly longer period of time, an average of $22 \mathrm{sec}$, examining the list of objects that were embedded within a pictorial scene. This was in contrast to an average of $8 \mathrm{sec}$ for poor imagers to examine the stimulus list. In essence, vivid imagers appeared to be storing the list of objects-to-be-found in longterm memory (LTM), whereas poor imagers appeared only to peruse the list.

In a related study, Wallace (1991) examined the ability of subjects to proofread a text for misspelled or mistyped words. Since proofreading is in essence a type of visual search, Wallace was concerned with the performance of subjects in this type of task compared with the more straightforward visual search for an embedded object.

Proofreading accuracy was examined on subjects who were judged to be vivid or poor imagers as assessed by the VVIQ. Six 1-page passages from various texts were prepared such that each contained 26 misspelled or mistyped words. The words were produced by removing a

Correspondence should be addressed to B. Wallace, Department of Psychology, Cleveland State University, Cleveland, OH 44115. middle-position letter and substituting another letter that was in shape either the same or different. Results indicated that subjects who were judged to be vivid imagers made fewer proofreading errors when the substitute letter was the same shape as the letter it replaced. This was not found to be the case when the substitute letter was different in shape.

In addition, strategy differences were noted in searching for the misspelled/mistyped words. Poor imagers reported that they tended to look for misspelled/mistyped words by examining the passage one word at a time. In essence, they appeared to be using Crawford and Allen's (1983) detail search strategy. Within each word, they would examine the letters contained therein and decide if the letters correctly spelled a word. However, the majority of the poor imagers reported that they did not really examine each letter within each word. Instead, they would examine the first letter or two and make a decision based on these letters. This, of course, is likely to lead to a failure to detect a misspelled/mistyped word. After performing a quasi-letter-by-letter analysis, they would determine if the word was spelled correctly and then move to the next word.

In performing the type of search described by poor imagers, it is also clear that their detail strategy could not make use of the imagery that would be required to find most errors in print. If they tended to examine only the first few letters in each word, it would generally not be easy to compare the image of a word held in LTM with the percept of the word being examined.

Vivid imagers also reported that they searched for misspelled/mistyped words in a word-by-word fashion. Unlike with poor imagers, however, a quasi-letter-by-letter search did not appear to be taking place. Instead, vivid imagers reported perusing each word as a whole and making a decision concerning spelling accuracy on the basis 
of this overall examination; they appeared to be using Crawford and Allen's (1983) holistic strategy. This included examining the spelling of the word within the context of the sentence. In essence, subjects examined the totality of letters within a word as fitting together. In doing so, it is more likely that subjects compared the percept of the word being examined with an image of the word in LTM. And since vivid imagers tended not to make a decision on the basis of the input of only a few letters, they made fewer errors and, as a consequence, identified more incorrect spelling.

Given that individual differences exist with respect to the performance of subjects in searching for an embedded letter (Wallace, 1991) or object (Wallace, 1988) within an array of other letters or objects, it is also possible that differences will emerge concerning the ability of subjects to process other tasks that require finding perceptual differences in an array of stimuli. One such task is reading EEG patterns. When a neurologist or technician examines EEG waveforms, it is important that this individual be able to find anomalies or differences within the stimulus array. After all, it is the ability to discern such differences from normality that provides the critical information for making a diagnosis.

If individual differences exist in a proofreading task and holistic processors perform the task differently from detail processors, such individual differences may also be true with respect to reading EEG waveforms. This is because there are interesting parallels between the reading of textual information and the reading of EEG data. For example, EEG recordings can be understood in terms of an information hierarchy similar to that for text. In text, individual strokes and shapes combine to produce letters. These in turn combine to produce words (concepts), which combine to produce phrases (ideas), sentences (thoughts), and so on. In EEGs, individual wavelets build up into complexes and sequences that form the parts of more complex events. These combine to create an electrophysiological picture that changes from moment to moment, reflecting the patient's overall brain state. In addition, while text reading includes an active component of building a conceptual framework and anticipating the most likely words and phrases, EEG reading also requires the development of a "clinical gestalt." Within this gestalt, waveform patterns are interpreted and particular features may be recognized or neglected and clinically accepted or rejected in terms of the working hypothesis.

Our overall view was that EEG readers would exhibit behavior that reflected a distribution along a spectrum of detailed, careful scrutiny on one extreme and a more holistic, hypothesis-based interpretation on the other. We expected individual physicians to fall generally within this range in a manner that reflected their individual styles.

\section{METHOD}

\section{Subjects}

Five male neurologists affiliated with the Cleveland Clinic Foundation served as volunteers. All individuals had extensive experience in reading EEG printouts and in making subsequent diagnoses concerning neurological anomalies, especially as related to forms of epilepsy.

\section{Procedure}

The subjects were individually tested. Prior to participation, the neurologists were informed that within a 4-week period, their performance in processing randomly selected EEG recordings would be recorded by a video camera. They were told that this recording would be used exclusively to examine how they processed EEG records and that an evaluative report concerning their efficiency and accuracy would remain confidential with the experimenters.

The subjects were instructed to read and interpret the record as they normally would and not to deviate from their usual activity. Within the confines of the working day and at their convenience, the subjects were asked to examine a record that had been placed on a table in a room used exclusively for routine EEG reviewing. Over this table (in the ceiling) was mounted the video camera for recording the activity of the subjects. In addition, the subjects were provided with the necessary tools for processing the EEG records (i.e., markers, rulers, and gauges).

Although the subjects were aware that a camera was mounted over the reading table, they were not aware of when the camera was recording since they were informed that it would not be continuously operating. In reality, the camera was always operating when a neurologist was in the EEG reading room.

In addition, all subjects completed the VVIQ. However, this administration was accomplished independently of the EEG experiment. Thus, the subjects were not aware that their performance on the VVIQ was affiliated with their other experimental participation.

\section{RESULTS}

The recorded tapes were examined by two judges. Each judge viewed each tape and recorded the length of time required to process an EEG printout, the number of pauses or rest periods required by the subjects, and the numbers and types of markings made on each printout and/or on a notepad. In addition, the judges observed the types of cognitive strategies used by the subjects in reading the printouts.

Of the 5 subjects, 3 demonstrated a holistic strategy for processing the EEG printouts. That is, these individuals spent significantly less time (corrected for the number of pages in the printout) processing an EEG record $[t(3)=$ $9.81, p<.01$ ]. They also exhibited fewer pauses during the reading of an EEG printout $[t(3)=4.77$, $p<.02$ ]. The typical strategy was to read the EEGs by rapidly flipping through the pages. When the reader spotted what appeared to be an anomalous EEG pattern, he would stop and make a note and/or measure the amplitude of a wave. After so doing, he would continue to rapidly flip through the printout.

In addition to the rapidity with which the aforementioned subjects processed an EEG readout, two additional strategies were noted. These individuals rarely flipped back to previous pages in the printout. They would occasionally refer back to the early pages of the printout, but they did not make a habit of flipping back and forth for comparisons. Also, these individuals made few notes and took few measurements during their processing of a printout. They would occasionally make a note or take a measurement, but this was the exception.

For the remaining subjects, who demonstrated what Crawford and Allen (1983) have labeled a detail strategy, 
processing EEG printouts was noticeably different. Instead of rapidly flipping through the pages, these individuals were much slower. They would spend much more time, on the average, per page compared with the subjects who demonstrated a holistic strategy $[8.5 \mathrm{sec}, S D=$ 1.8 , vs. $2.3 \mathrm{sec}, S D=0.4, t(3)=18.61, p<.001]$. In addition, they took more notes [6.5 vs. $2.7, t(3)=$ $4.36, p<.05]$ and made more measurements [4.3 vs. $1.0, t(3)=5.93, p<.01]$ per printout. These individuals would also more often refer back to previous pages to make comparisons $[7.0$ vs. $2.0, t(3)=15.19$, $p<.001]$. In essence, their behavior was marked by frequent back-and-forth flipping to compare and recompare EEG patterns and potential anomalies.

Furthermore, although only 5 subjects participated in this study, a significant relationship was found between type of strategy used in processing EEG printouts and imagery vividness as established by the VVIQ. Holistic processing was associated with a higher score on the VVIQ $\left[r_{\mathrm{pb}}(3)=.88, p<.05\right]$.

Finally, averaged over the different dependent measures, interjudge reliability was significant and strong $\left(r_{\mathrm{pb}}=.99, p<.001\right)$. There were virtually no differences between judges in assessing the performances of the subjects.

\section{DISCUSSION}

Despite the fact that only 5 subjects were used in the present study, it is clear that individual differences exist in the reading of EEG records. Generally, two types of strategies were evidenced here. These were the detail strategy and the holistic strategy (see Crawford, 1979, 1981; Crawford \& Allen, 1983). The former utilizes a more meticulous and slower examination of EEG patterns; the latter exhibits a faster examination. And, those who utilize the detail strategy appear to be slower because they do not utilize the same degree of imagery in the processing of information as holistic processors and, as a result, do not store information in LTM for use in reading EEG patterns. Also, although we did not verify the accuracy of the EEG diagnoses by the volunteer neurologists, it is unlikely that accuracy was affected by type of strategy employed. After all, the 5 neurologists were all experienced EEG readers.
Given that two types of strategies are evident in the reading of EEG printouts, there are important implications for future applications in this field. A general trend is the complete elimination of paper in the recording of EEG activity. This is possible through the use of computers for the recording and display of EEG patterns. It is important to identify and understand individual differences in the reading and processing of such patterns. For example, if some readers are slow and others are fast or if some use a detail strategy and others use a holistic strategy, this has implications for the rate at which EEG patterns can be displayed and for potential aids that must be provided to readers so that accuracy of reading and interpretation is not affected. In essence, if we understand the information-processing parameters involved in reading EEG patterns, these parameters can be incorporated into future work environments where EEG activity is being studied.

While the present study has identified one source of variability (information-processing strategy) in the reading of EEG activity, there are undoubtedly many more. These need to be examined and incorporated into future applications as we move from a paper environment for recording EEG activity to one that is completely paperless with reliance on the use of computers (see Collura, Braun, Jacobs, Burgess, \& Turnbull, 1991).

\section{REFERENCES}

Collura, T. F., Braun, D. S., Jacobs, E. C., Burgess, R. C., \& Turnbull, J. P. (1991, November). A work station-based viewer for paperless clinical EEG. Paper presented at the annual meeting of the IEEE Engineering in Medicine and Biology Society, Orlando, FL.

CraWFord, H. J. (1979, October). Can hypnosis enhance visual imagery processing and memory? Paper presented at the annual meeting of the Society for Clinical and Experimental Hypnosis, Denver, CO.

CraWford, H. J. (1981). Hypnotic susceptibility as related to gestalt closure tasks. Journal of Personality \& Social Psychology, 40, 376-383.

Crawford, H. J., \& Allen, S. N. (1983). Enhanced visual memory during hypnosis as mediated by hypnotic responsiveness and cognitive strategies. Journal of Experimental Psychology: General, 112, 662-685.

MARKS, D. F. (1973). Visual imagery differences in the recall of pictures. British Journal of Psychology, 64, 17-24.

WALLACE, B. (1988). Imaging ability, visual search strategies, and the unvividness paradox. Journal of Mental Imagery, 12, 173-184.

WALLACE, B. (1991). Hypnotic susceptibility, imaging ability, and information processing: An integrative look. In R. G. Kunzendorf (Ed.), Mental imagery (pp. 89-100). New York: Plenum.

(Manuscript received June 24, 1992.) 